\title{
Alveolar Bone Preservation Using an Orthodontic Mini-implant in an Adolescent Patient with Multiple Missing Teeth
}

\author{
Bum-Jun Lee', Young-Taek Kim, DDS, MSD, Ph.D², Jin-Joo Yoo, DDS, Ph.D², Ji-Yeon Lee DDS, \\ MSD, Ph. D* $^{4 *}$ \\ ${ }^{1}$ Resident, Department of Orthodontics, National Health Insurance Service Ilsan Hospital, Goyang-city, Korea \\ ${ }^{2}$ Associate Professor, Department of Periodontics, National Health Insurance Service llsan Hospital, Goyang-city, \\ Korea \\ ${ }^{3}$ Assistant Professor, Department of Prosthdontics, National Health Insurance Service Ilsan Hospital, Goyang- \\ city, Korea \\ ${ }^{4}$ Professor, Department of Orthodontics, National Health Insurance Service Ilsan Hospital, Goyang-city, Korea \\ *Corresponding author: Ji-Yeon Lee, Department of Orthodontics, National Health Insurance Service Ilsan \\ Hospital, 100 Ilsan ro, Ilsan donggu, Goyang-city, Gyeonggi do 10444, Korea \\ Tel : +82-31-900-0629. eFax : +82-303-3448-7138. E-mail : jylortho@nhimc.or.kr
}

OPEN ACCESS

pISSN 2765-7833

eISSN 2765-7841

Journal of implantology and applied sciences 2021; 25(3): 124-131

https://doi.org/10.32542/implantology.2021013

Received: March 2, 2021

Revised: June 21, 2021

Accepted: August 23, 2021

\section{ORCID}

Bum-Jun Lee

https://orcid.org/0000-0003-0204-8529

Young-Taek Kim

https://orcid.org/0000-0002-5132-5783

Jin-Joo Yoo

https://orcid.org/0000-0001-9346-723X

Ji-Yeon Lee

https://orcid.org/0000-0002-4738-1184

Copyright $\odot$ 2021. The Korean Academy of Oral \& Maxillofacial Implantology

(7) This is an Open Access article distributed under the terms of the Creative Commons Attribution Non-Commercial License (http://creativecommons. org/licenses/by-nc/4.0/) which permits unrestricted non-commercial use, distribution, and reproduction in any medium, provided the original work is properly cited.

\section{Abstract}

After tooth extraction, the alveolar ridge undergoes a series of structural changes, including the inevitable vertical and horizontal loss of alveolar bone. In this case, growth modification was performed on an 11-year-old patient with skeletal Class II malocclusion with multiple congenitally missing teeth and early loss of a deciduous tooth without succedaneous replacement using an intraoral orthopedic appliance. The space from the lost deciduous teeth was effectively maintained. At the age of 14 years, an orthodontic mini-implant with a diameter and length of 1.6 and $7 \mathrm{~mm}$, respectively, was inserted into the alveolar bone to prevent additional alveolar bone resorption and maintain alveolar bone height until growth completion. At the age of 18 years, the second phase of orthodontic treatment was started to properly set the time of dental implant placement and the completion of orthodontic treatment. At the age of 19 years, the alveolar bone height was properly maintained until dental implant placement. The dental implant was successfully placed without additional vertical bone augmentation. Therefore, we successfully preserved the alveolar bone by inserting an orthodontic mini-implant into the site of early deciduous tooth loss without succedaneous replacement in an adolescent patient.

Keywords: Adolescent patient, Alveolar bone preservation, Orthodontic mini-implant

\section{I . Introduction}

After tooth extraction, the alveolar ridge structure changes through a series of healing processes that cause alveolar bone resorption. According to Schorpp, ${ }^{1}$ two-thirds of the alveolar bone is absorbed within three months after tooth extraction. Thereafter, the alveolar width is reduced to 50\% (5-7 mm) within 12 months. Additionally, after deciduous 
molar extraction, the alveolar width decreases by $25 \%$ in the first four years, and $5 \%$ more in the next three years. ${ }^{2}$ Alveolar bone resorption can be prevented through ridge preservation after permanent tooth extraction. ${ }^{3}$

However, when performing prosthetic procedures using periodontal surgery and dental implant placement for alveolar bone preservation in adolescent patients, residual growth of the alveolar bone causes prosthetic infraocclusion, which is disadvantageous to occlusion formation. Therefore, prostheses with dental implant placement during adolescence must be postponed until growth is complete. ${ }^{4}$

In adolescent patients with congenital permanent tooth loss, maximal preservation of the deciduous tooth without a succedaneous tooth is optimal for the maintenance of alveolar bone until the completion of growth. If the tooth is extracted, the prosthesis placement should be postponed until after the completion of growth, and proper space maintenance and alveolar bone preservation are essential. ${ }^{5}$ Here, we report the successful prevention of alveolar bone resorption in an adolescent patient who lost a deciduous tooth without a succedaneous tooth using an orthodontic mini-implant.

\section{II . Case Report}

\section{Etiology and diagnosis}

An 11-year-old male presented with a chief complaint of achieving a well-balanced maxilla, mandible, and even dentition. He had a history of tonsillectomy but no dental history. Intraoral photographs showed
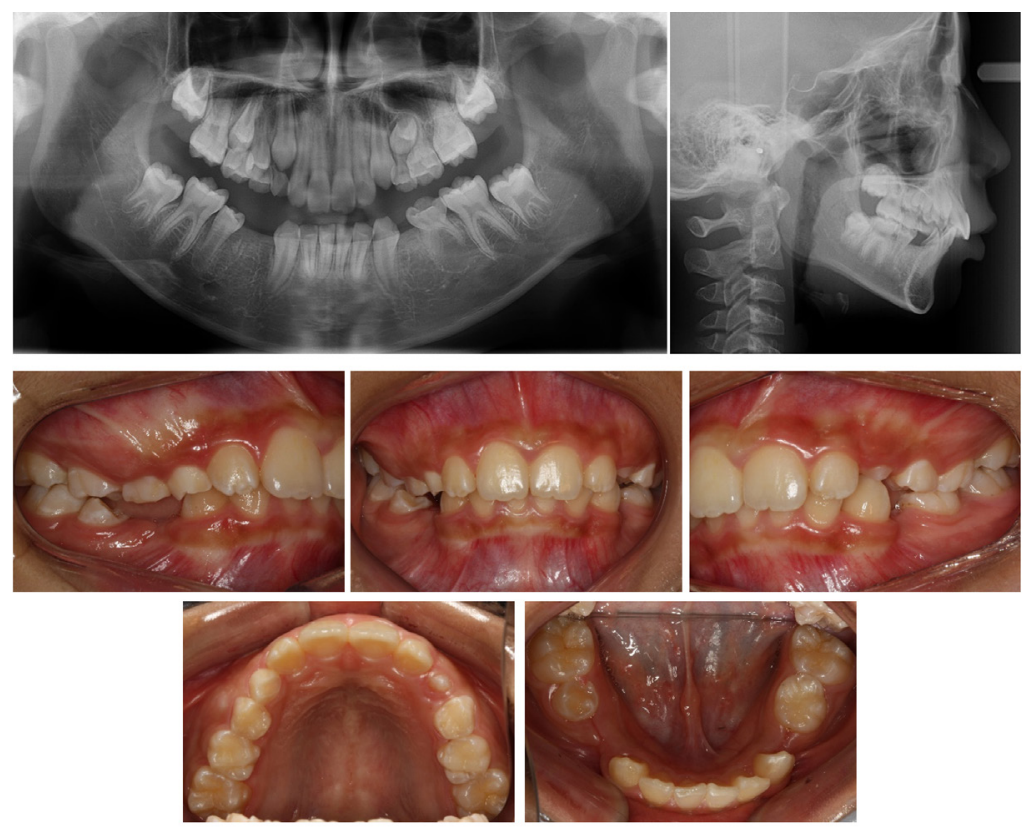

Fig. 1. Pre-treatment panoramic, lateral cephalometric radiographs and intraoral photographs (11 years old). 
that both mandibular deciduous first molars were extracted, and a bilateral Class II molar key was observed. Panoramic and lateral cephalometric radiographs showed the congenital absence of the maxillary left second premolar, the mandibular left first and second premolars and the mandibular right first premolar, and a residual root of the mandibular right deciduous first molar. The maxillary left deciduous second molar and mandibular left deciduous second molar remained (Fig. 1).

Therefore, we diagnosed the patient with skeletal Class II malocclusion with multiple congenitally missing teeth. The main treatment objective was to obtain normal occlusion and improve esthetics by maintaining the space of the missing tooth and growth modification. Due to skeletal Class II malocclusion, we planned to use an intraoral orthopedic appliance (Class II activator) to promote mandibular growth and maintain the space where deciduous teeth were lost early. In addition, the sites of congenitally missing permanent teeth were planned for prosthetic dental implant placement after the growth was completed.

\section{Treatment progress}

From the age of 11 to 12 years, the patient was actively treated with an intraoral orthopedic appliance, followed by a maintenance period up to the age of 13 years and 6 months. Proper space maintenance of the congenitally missing permanent teeth was observed in a panoramic, lateral cephalometric radiograph, and intraoral photographs at the age of 13 years and 6 months (Fig. 2). At the age of 14 years, an
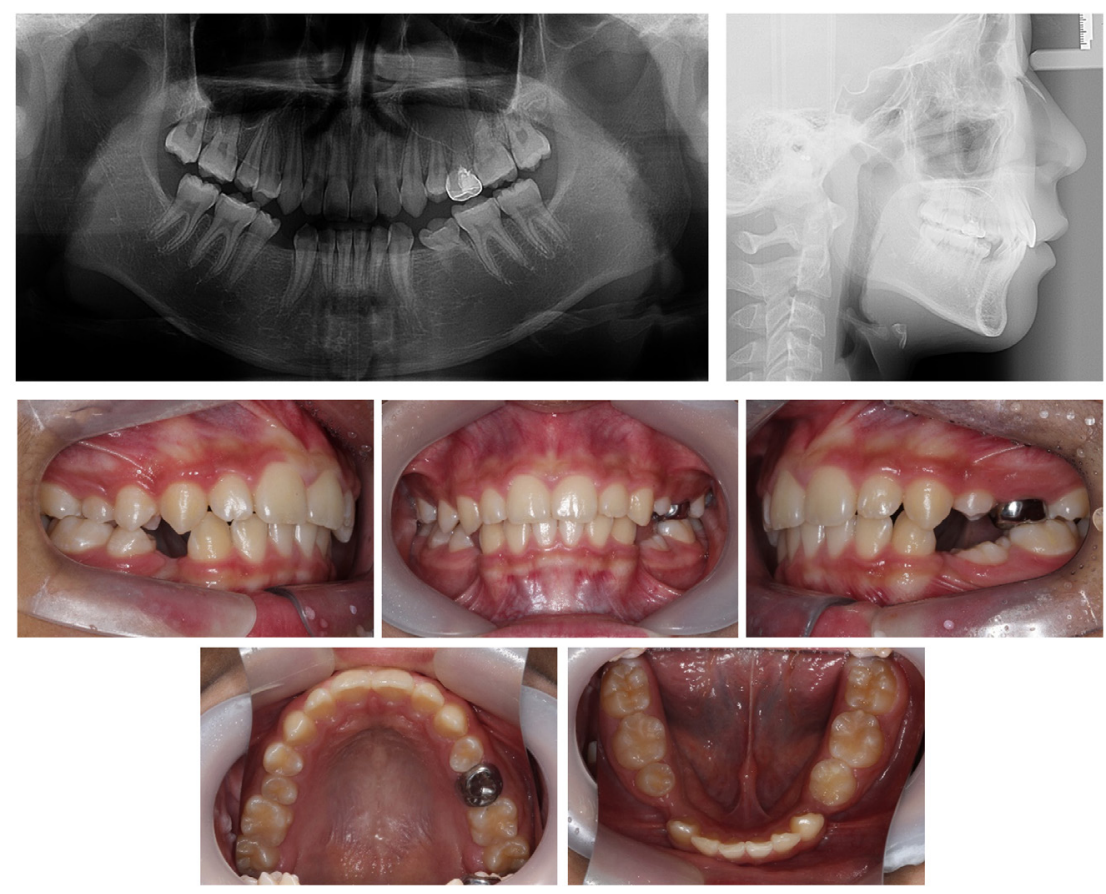

Fig. 2. Panoramic, lateral cephalometric radiographs and intraoral photographs after growth modification ( 13 years and 6 months old). 
orthodontic mini-implant of $1.6 \mathrm{~mm}$ in diameter and $7 \mathrm{~mm}$ in length (ORLUS ET; Ortholution Co., Seoul, Korea) was inserted into the alveolar ridge of the mandibular right first premolar to prevent additional alveolar bone resorption. The distance from the alveolar crest to the tip of the mini-implant, measured by ImageJ (ver. 1.53i, National Institutes of Health, Bethesda, USA), at the time of implantation was $3.27 \mathrm{~mm}$, and only $0.19 \mathrm{~mm}$ of bone resorption was observed within 24 months (Fig. 3).

At the age of 17 years, the mandibular left deciduous second molar was maintained, but the patient returned to the hospital after maxillary left deciduous second molar extraction due to dental caries. At 18 years of age, we started the second phase of orthodontic treatment with a fixed orthodontic appliance to properly set the time of dental implant placement and completion of orthodontic treatment. Intraoral photographs and panoramic radiograph showed that the proper space of the sites of the congenitally missing permanent teeth was stably maintained, and a normal skeletal growth pattern was observed on the lateral cephalometric radiograph (Fig. 4). Therefore, we decided to perform prosthodontic treatment through dental implant placement at the sites of the congenitally missing permanent teeth as we planned.

Twelve months after the second phase of orthodontic treatment, the Class I canine and molar key was formed, with maintenance of the appropriate space for the congenitally missing permanent teeth. Additionally, 10 mm-long dental implants (SuperLine ${ }^{\circledR}$, Dentium, Seoul, Korea) was successfully

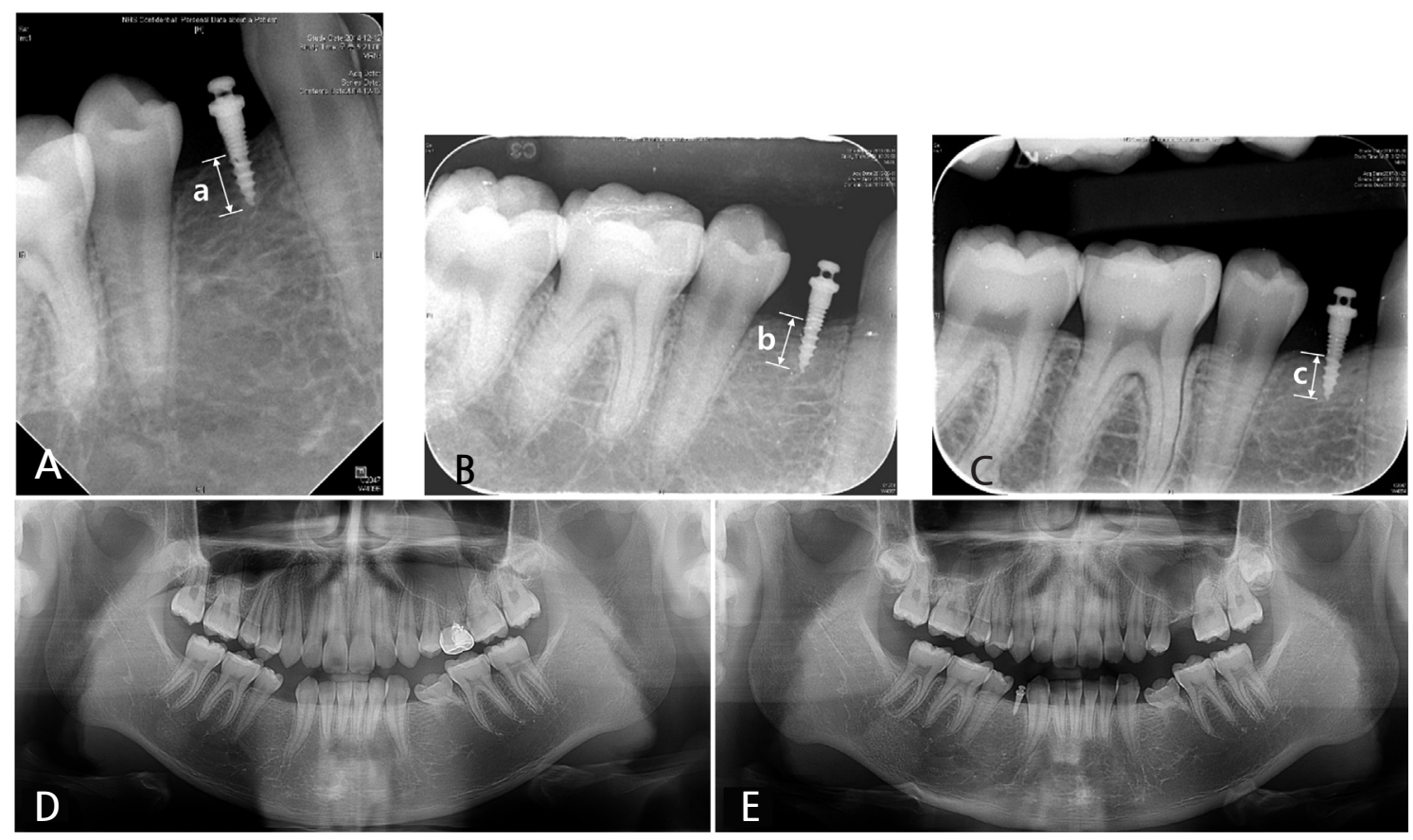

Fig. 3. The distance between the lowest point of the alveolar crest and the tip of the mini-implant was measured parallel to the long axis of the mini-implant. Inserted mini-implant was used as a reference and all images were calibrated by ImageJ software program. (A) The first day of mini-implant insertion $(a=3.27 \mathrm{~mm})$, (B) After 18 months $(b=3.22 \mathrm{~mm})$, (C) After 24 months $(c=3.08 \mathrm{~mm})$, (D)Comparison of panoramic radiographs right before orthodontic mini-implant insertion, (E) After 24 months. 
placed into all missing teeth areas (Fig. 5). After all prostheses were completed, the fixed orthodontic appliances were removed and lingual fixed retainers were bonded from canine to canine in both arches at 5 months after all fixtures were installed (Fig. 6).
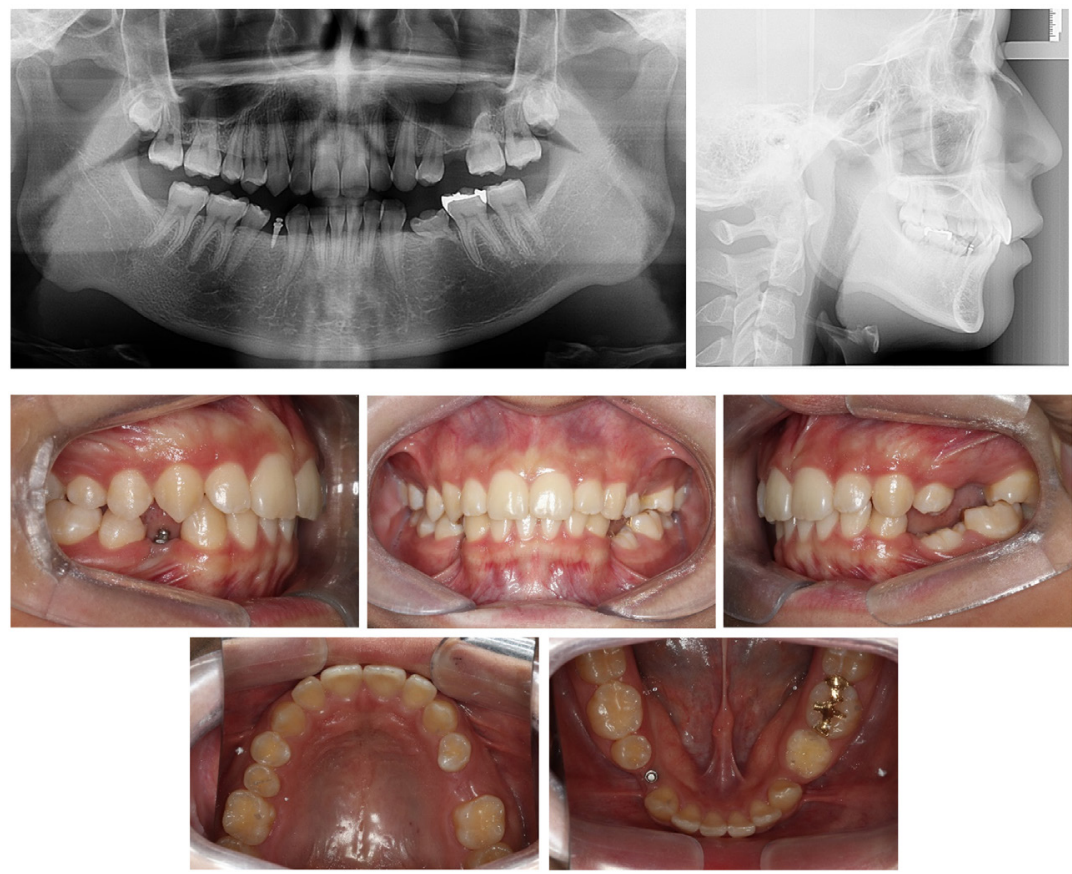

Fig. 4. Panoramic, lateral cephalometric radiographs and intraoral photographs after growth completion (18 years old).
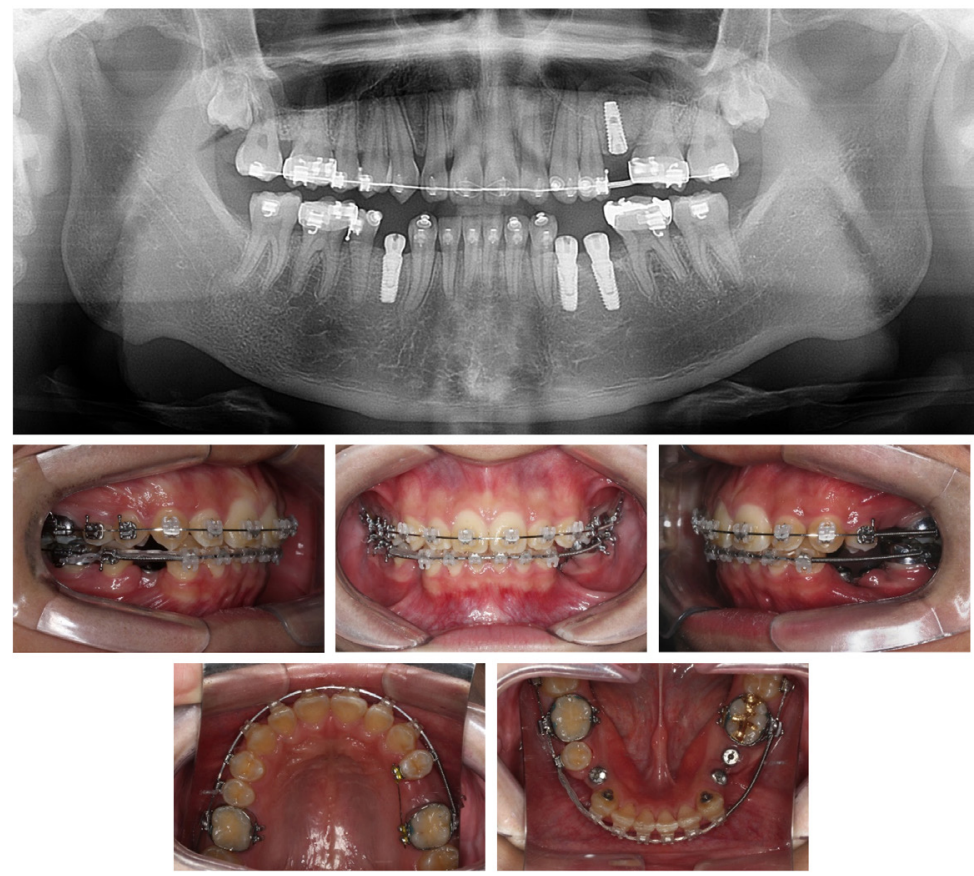

Fig. 5. Panoramic radiograph and intraoral photographs after implant placement (19 years old). 

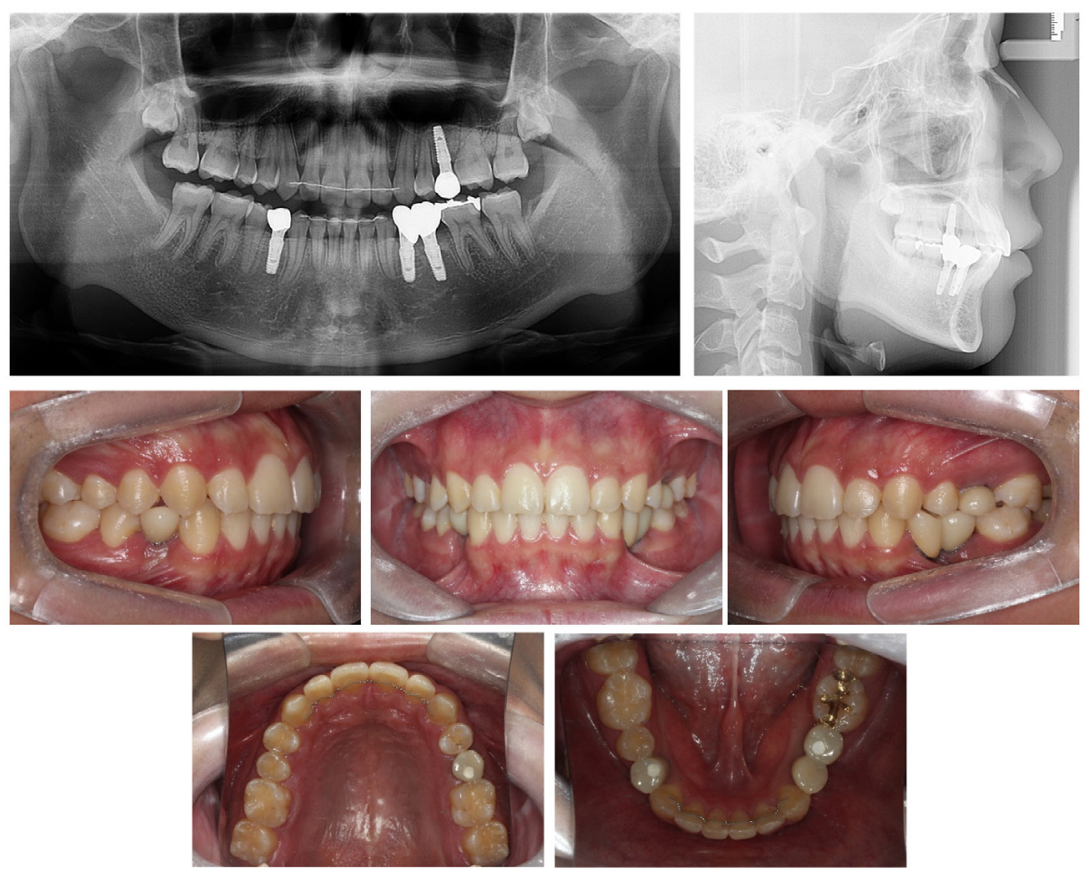

Fig. 6. Post-treatment panoramic, lateral cephalometric radiographs and intraoral photographs.

\section{III . Discussion}

Congenitally missing permanent teeth cause various esthetic, physiological, and functional problems such as malocclusion, periodontal disease, decreased growth of alveolar bone, and problems such as the loss of space and eruption of the opposing tooth. Therefore, maintaining deciduous teeth in adolescent patients with multiple congenitally missing permanent teeth is the ideal condition for proper occlusion after growth is complete. However, the loss of a deciduous tooth without a succedaneous tooth requires alternatives and proper management. ${ }^{5,6}$

The first treatment option is orthodontic space closure of the site of the missing tooth, and the second is the maintenance of space for prosthetic treatment. ${ }^{7}$ Prosthetic treatment with dental implant placement in the growing adolescent is contraindicated because the growth of the alveolar bone causes dental implant prosthesis infraocclusion. ${ }^{8}$ In the first option, the progress of space closure can be performed through skeletal and soft-tissue analysis when facial esthetics and occlusion are expected to improve. However, space closure should not be performed if the results might be unfavorable for esthetics and occlusion. $^{9}$

In the second option, the alveolar bone is absorbed in the vertical and horizontal directions after tooth extraction. Clinically, bone resorption was observed with a width of $3.87 \mathrm{~mm}$ and a height of $1.67-2.03$ $\mathrm{mm}^{10}$ after tooth extraction. After the growth was completed, the bone width was augmented by placing 
a dental implant with a ridge split and bone graft. However, the loss of alveolar bone height is irreversible, and dental implant placement at the site of low alveolar bone height is very difficult and unfavorable to oral hygiene and esthetics; therefore, the prognosis of dental implant is poor. ${ }^{11}$ Therefore, maintaining alveolar bone height is essential. When ridge preservation is performed with a bone graft and resorbable membrane at the time of tooth extraction to prevent the resorption of alveolar bone reduced resorption of alveolar bone of $1.72 \mathrm{~mm}$ vertically and $2.19 \mathrm{~mm}$ buccolingually can be expected. ${ }^{12}$

Furthermore, as a method of overcoming the vertical loss of alveolar bone after tooth extraction, autogenous onlay bone grafts (AOBGs) have been performed on bone defects in adult patients to ensure successful dental implant placement, which resulted in bone formation and a high success rate. ${ }^{13}$ A high dental implant success rate was also reported when a dental implant was placed after distraction osteogenesis (DO) at the site of severe bone resorption. ${ }^{14}$ Both techniques are effective for vertical bone reconstruction, but AOBGs have a risk of morbidity at the donor site and nerve damage, and DO has a risk of bone fracture, device damage, and bone resorption. Moreover, both methods are invasive and have a relatively large surgical range. ${ }^{15}$

According to a systemic review by Bidra, when a mini-implant was placed in the edentulous region, the marginal bone appeared mature and healthy without additional bone loss. The advantages of this procedure are flapless surgery and a rare association with bone grafting procedures. The insertion torque for mini-implant placement is significantly lower than that used for standard dental implants, and miniimplants can be placed at any stage of treatment, with low cost and few patient-related factors. When used for interim fixed and removable prosthodontic treatment, tangible treatment outcomes include immediate satisfaction for patients. ${ }^{16}$

In this case, when the patient was 14 years old, an orthodontic mini-implant with a diameter of $1.6 \mathrm{~mm}$ and length of $7 \mathrm{~mm}$ was placed on the alveolar ridge to prevent alveolar bone resorption at the site of the mandibular right first premolar. For 24 months after mini-implant implantation, only $0.19 \mathrm{~mm}$ of vertical resorption of alveolar bone was observed, maintaining good alveolar bone height in the patient up to 19 years of age.

\section{IV . Conclusion}

Maintaining the alveolar bone at the congenitally missing permanent tooth site in growing patients is essential for successful dental implant placement and prosthesis in the future. In this case, an orthodontic mini-implant was inserted into the alveolar ridge to preserve the alveolar bone height in an adolescent with early loss of a deciduous tooth without a succedaneous tooth. After growth completion, the dental implant was appropriately placed without additional vertical bone augmentation. 


\section{References}

1. Schropp L, Wenzel A, Kostopoulos L, Karring T. Bone healing and soft tissue contour changes following single-tooth extraction: a clinical and radiographic 12-month prospective study. Int $\mathrm{J}$ Periodontics Restorative Dent 2003;23:313-23.

2. Ostler MS, Kokich VG. Alveolar ridge changes in patients congenitally missing mandibular second premolars. J Prosthet Dent 1994;71:144-9.

3. Lindhe J, Cecchinato D, Donati M, Tomasi C, Liljenberg B. Ridge preservation with the use of deproteinized bovine bone mineral. Clin Oral Impl Res 2014;25:786-90.

4. Thilander B, Ödman J, Gröteborg K, Friberg B. Osseointegrated implants in adolescents. An alternative in replacing missing teeth? Eur J Orthod 1994;16:84-95.

5. Kokich VG, Kokich VO. Congenitally missing mandibular second premolars: clinical options. Am J Orthod Dentofac Orthop 2006;130:437-44.

6. Miller TE. Implications of congenitally missing teeth: Orthodontic and restorative procedures in the adult patient. J Prosthet Dent 1995;73:115-22.

7. Jha P, Jha M. Management of congenitally missing second premolars in a growing child. J Conserv Dent 2012;15:187-90.

8. Bernard J, Schatz J, Christou P, Belser U, Kiliaridis S. Long-term vertical changes of the anterior maxillary teeth adjacent to single implants in young and mature adults: A retrospective study. J Clin Periodontol 2004;31:1024-8.

9. Northway WM. The nuts and bolts of hemisection treatment: managing congenitally missing mandibular second premolars. Am J Orthod Dentofac Orthop 2005;127:606-10.

10. Van der Weijden F, Dell'Acqua F, Slot DE. Alveolar bone dimensional changes of post-extraction sockets in humans: a systematic review. J Clin Periodontol 2009;36:1048-58.

11. Agabiti I, Botticelli D. Two-stage ridge split at narrow alveolar mandibular bone ridges. J Oral Maxillofac Surg 2017;75:2115.e1-e12.

12. Troiano G, Zhurakivska K, Lo Muzio L, Laino L, Cicciù M, Lo Russo L. Combination of bone graft and resorbable membrane for alveolar ridge preservation: A systematic review, meta-analysis, and trial sequential analysis. J Periodontal 2018;89:46-57.

13. Iizuka T, Smolka W, Hallermann W, Mericske-Stern R. Extensive augmentation of the alveolar ridge using autogenous calvarial split bone grafts for dental rehabilitation. Clin Oral Implants Res 2004; 15:607-15.

14. Cheung LK, Chua HDP, Hariri F, Pow EH, Zheng L. Alveolar distraction osteogenesis for dental implant rehabilitation following fibular reconstruction: a case series. J Oral Maxillofac Surg 2013;71:255-71.

15. Zhao K, Wang F, Huang W, Wang X, Wu Y. Comparison of dental implant performance following vertical alveolar bone augmentation with alveolar distraction osteogenesis or autogenous onlay bone grafts: a retrospective cohort study. J Oral Maxillofac Surg 2017;75:2099-114.

16. Bidra AS, Almas K. Mini implants for definitive prosthodontic treatment: a systematic review. J Prosthet Dent 2013;109:156-64. 